\title{
Telemental Health Use and Refugee Mental Health Providers Following COVID-19 Pandemic
}

\author{
Lindsey Disney ${ }^{1}\left[\right.$ OD $\cdot$ Orion Mowbray ${ }^{2} \cdot$ Dana Evans $^{3}$ \\ Accepted: 6 April 2021 / Published online: 3 May 2021 \\ (c) The Author(s), under exclusive licence to Springer Science+Business Media, LLC, part of Springer Nature 2021
}

\begin{abstract}
The outbreak of COVID-19 has increased stress, fear, and anxiety for many people. At the same time, social isolation restrictions have disrupted most in-person mental health services. Many mental health providers are adapting to the crisis by utilizing telemental health. However, the literature is scant about how to most effectively utilize telemental health practices with refugee clients, many of whom do not speak English and require an interpreter, may have limited technological proficiency or access, and/or have additional case management needs and coordination as part of their treatment plan. The purpose of this study is to understand how mental health clinicians and case workers at a refugee-serving mental health clinic are successfully shifting their face-to-face practice to telemental health, the obstacles they encounter, and what resources they have found to be helpful. Two main themes emerged from the data: (1) refugee mental health providers display initiative and flexibility in their adaptation to telemental health and (2) providers reported numerous obstacles to effective telemental health, including client barriers, tech barriers, communication issues, and the challenge of reading nonverbal cues virtually. By better understanding telemental health when working with refugees, clinical social workers will be more effective in meeting the needs of a population with significant mental health needs and limited mental healthcare access.
\end{abstract}

Keywords Refugee $\cdot$ COVID-19 $\cdot$ Telemental health $\cdot$ Mental health practitioners $\cdot$ Qualitative

\section{Introduction}

The outbreak of coronavirus disease 2019 (COVID-19) has increased stress, fear, and anxiety for many people (Kirzinger et al., 2020; World Health Organization, 2020; Zhou et al., 2020). At the same time, social isolation restrictions have disrupted most traditional in-person mental health services. Many mental health providers are adapting to the crisis by utilizing telemental health (Zhou et al., 2020). Telemental health has been used since the 1960s (Field, 1996) across a wide variety of populations including children (North, 2020), the aging (Gellis et al., 2014), veterans (Levy et al., 2018; Tuerk et al., 2010), diverse racial and ethnic groups

Lindsey Disney

ldisney@albany.edu

1 School of Social Welfare, University at Albany, Albany, NY, USA

2 School of Social Work, University of Georgia, Athens, GA, USA

3 Positive Growth, Inc., Clarkston, GA, USA
(Stewart et al., 2017), those with comorbid physical health conditions (Cox et al., 2017), and rural populations (Levy et al., 2018). Evidence suggests that telemental health can be as effective at treating Post Traumatic Stress Disorder (Tuerk et al., 2010) and Major Depressive Disorder (Gellis et al., 2014) as in-person clinical services, the two disorders most prevalent in resettled refugees (Fazel et al., 2005).

The client benefits of telemental health include feasibility, increased satisfaction, and lowered anxiety related to initiating treatment (Tuerk et al., 2010; Gros et al., 2018). Additionally, telemental health as an alternative to in-person services can ameliorate certain barriers to healthcare access, such as lack of transportation, linguistically appropriate services, or services in an individual's area (Stewart et al., 2017). At the same time, there have been multiple obstacles reported with providing mental health treatment via telemental health, including resistance from clients and clinicians, lack of training, clinical workflow and technology barriers, licensure or credentialing requirements, reimbursement barriers, and increased administrative overhead (Brooks et al., 2013; Perry et al., 2020; Standing et al., 2018). 
Despite a substantial body of knowledge related to telemental health (Standing et al., 2018), there is scant evidence about telemental healthcare practices specifically with resettled refugee clients (Ashfaq et al., 2020). Resettled refugees have high rates of Post Traumatic Stress Disorder (PTSD) and Major Depressive Disorder (MDD) (Lamkaddem et al., 2014). Additionally, they are less likely to utilize mental healthcare services due to cultural and structural barriers (Lamkaddem et al., 2014), potentially making them a particularly vulnerable population during a global pandemic. Additionally, many resettled refugees do not speak English and require an interpreter for therapy sessions. Refugees may also have difficulty accessing or using the technology needed for telemental healthcare, and/ or may have case management needs as part of their treatment plan, which can impede mental health providers' ability to successfully deliver telemental health services.

Research on telemental health with refugee clients, displaced or resettled, is limited, despite a recognition in the literature that telemental health has the potential to address some mental healthcare disparities (Almoshmosh et al., 2020; Ashfaq et al., 2020; Soron et al., 2019). In fact, in a systematic review of telemental health with Arab refugees over a twenty-year period, only seven articles were identified (Ashfaq et al., 2020). Within the handful of available studies is Jefee-Bahloul et al.'s study (2014) on the acceptance of telepsychiatry in Syrian refugee camps, which found that although approximately $40 \%$ of Syrian refugees screened positive for PTSD, only $15 \%$ were open to telepsychiatry (Jefee-Bahloul et al., 2014). Another refugee telemental health study examined resettled refugees' opinions on inperson, video, or phone interpreters, with a clear preference for in-person interpreters to video interpreters (although widely accepting both), and video interpreters to phone interpreters reported by refugee clients (Schulz et al., 2015).

Given the prior research on telemental health and the scarce literature available to inform telemental health with refuge populations, the purpose of this study is to understand how mental health clinicians and case workers at a refugeeserving mental health clinic are successfully shifting their face-to-face practice to telemental health, the obstacles they encounter, and what resources they have found to be helpful. By better understanding telemental health use when working with refugees, clinical social workers can better serve this population with significant mental health needs and limited access to mental healthcare.

\section{Methods}

\section{Selection of Participants and Setting}

Participants were recruited from a refugee-serving outpatient mental health clinic in a large refugee resettlement area.
Some clinicians and case managers work exclusively with either refugee clients or non-refugee clients, and some serve both populations. This survey was sent to all mental health providers employed at the agency $(\mathrm{N}=42)$, with a response rate of $81.0 \%(\mathrm{~N}=34)$. Participants who did not serve refugee clients were excluded from this study, resulting in a final sample of 17 participants.

Consistent with previously obtained data on refugees seeking mental health services (Fazel et al., 2005), the majority of refugee clients at this clinic presented with Post Traumatic Stress Disorder related to trauma histories and Major Depressive Disorder related to resettlement. Given the multiple logistical stressors in a resettled refugee's life (i.e. financial, housing, and employment instability, learning English, identifying and accessing social services, navigating new systems such as school, medical, legal and transportation), case management is a vital component to the treatment plan for the majority of refugee clients. In order to provide culturally sensitive mental health treatment, this clinic utilizes an integrated treatment model that allows therapists to provide case management themselves, and for case managers who are licensed therapists to also provide therapy. Case managers who are not licensed therapists receive training in mental health diagnosis, treatment, and coping skill development, and are often members of the local refugee or immigrant communities themselves. The majority of refugee clients at this clinic are from Afghanistan, Bhutan, Burma, Congo, Iraq, Somalia, and Syria. Some clients are new arrivals and others have lived in the U.S. for many years.

The lead author for this study has worked at this clinic for nine years in a direct clinical practice position. The third author also works for this clinic as the clinical director. As both practitioners and researchers, we were familiar with the barriers that the refugee population faces in accessing mental health treatment, and as the COVID-19 pandemic began to unfold, we observed a need in the published literature and our knowledge base about what additional barriers treatment providers might face in providing services to their clients via telemental health. Concurrently, in March 2020, the Georgia Department of Behavior Health and Developmental Disabilities (DBHDD) waived the credentialing requirements to practice telemental health and prohibited in-person services, causing many mental health professionals at this clinic, as well as throughout the state, to pivot quickly to telemental health. The present study examines this phenomenon through qualitative, electronic interviews with participants, examining their experiences in the delivery of telemental health services to refugee clients. 


\section{Data Collection Methods and Instruments}

Data was collected from an online survey created in Qualtrics and sent via email to participants. Recruitment occurred through respondents' place of employment. All participants were informed about their confidentiality and were asked to voluntarily electronically consent to participate. There was no incentive for participation. The institutional review board of University of Georgia approved all procedures. The survey was open for 25 days in May 2020, and participants were contacted via email three times to fill out the survey.

The survey contained questions aimed at understanding participants' telemental health training and experiences prior to and during the COVID-19 pandemic. Qualitative questions focused on the changes made, difficulties encountered, and resources used in adapting to telemental health. See Table 1 for a full list of the interview questions that were asked. Participants also completed information on their background/demographics, including age, race, gender, and primary job title, and answered some short questions about their prior experiences and familiarity using telemental health.

\section{Data Analysis}

For this study, a thematic analysis was used to examine the electronic interview questions. Coding was done by hand, and the overall coding process utilized was "theming" the data, as defined by Saldaña (2015). The initial round of coding sought out verbatim words and phrases that provided an understanding of how the participants experienced telemental health (Creswell, 2012; Saldaña, 2015). The second round of coding served the analytic purposes of identifying overarching themes and subthemes. All coding was completed initially by the lead author of this study. Following the first round of coding, others from the author team reviewed the coding, provided feedback, and mutually agreed on a final coding process.

\section{Results}

\section{Participant Characteristics}

Participants were mostly female $(87 \%)$, and their ages ranged from 27 to 65 years old with a mean of 37 years old. Approximately half of participants identified as White (47\%), one-third as African American (33\%), 7\% as Asian, and $13 \%$ preferred not to answer. Participants identified their primary job title as: Therapist (47\%), case managers (29\%), psychologist (6\%) and advanced practice registered nurse (APRN) (6\%). Participants were asked to report their primary job title because at this agency, some therapists also provide case management, and some case managers also provide therapy. Fifteen of the 17 participants answered the demographic questions on the survey.

When asked about telemental health training, $41 \%$ of participants stated that they did have telemental health training prior to COVID-19; however, only $29 \%$ of participants had used telemental health prior to COVID-19. In regard to the type of telemental health used, participants reported using phone only (18\%), phone primarily and some video (47\%), phone and video equally (29\%), and video primarily and some phone $(6 \%)$. No one reported using only video-based telemental health.

Although the majority of participants (53\%) did not feel equipped to use telemental health prior to COVID-19, all participants reported they felt equipped to use telemental health at the time of the study. At the same time, when asked how much they agreed with the statement, "I am able to help my client as much using telemental health as using in-person sessions," only $75 \%$ strongly or somewhat agreed.

All participants reported they either strongly agreed (82\%) or somewhat agreed (18\%) with the statement "I am supported by my employer to adapt to telemental health." When asked what resources were helpful in adapting to telemental health, participants agreed that telemental health trainings (94\%), electronic resources (i.e. email, listserv, internet search) (94\%), and periodic check-ins with supervisors on a weekly or biweekly basis (88\%) were helpful.
Table 1 Qualitative interview questions
1. What have you found to be helpful in adapting to telehealth?

2. Please describe 3 changes you made to be able to do your work successfully using telehealth

3. Please describe 3 difficulties you have had in using telehealth

4. Please describe 3 available resources that provide assistance in using telehealth that you have used

5. Is there anything else that could be helpful to adopting telehealth practices? 


\section{Telemental Health Themes Among Refugee Mental Health Providers}

Participants presented a picture of both adaptation and difficulty with the adoption of telemental health for service delivery. Two main themes emerged from the data: (1) refugee mental health providers take initiative and are flexible in their adaption to telemental health and (2) refugee mental health providers reported numerous obstacles to effective telemental health, including client barriers, technological difficulties, language issues, and the lack of nonverbal cues. Each theme had two subthemes, as shown below. Table 2 displays telemental health themes among refugee mental health providers and exemplar quotes.

Theme 1: Refugee Mental Health Providers Display Initiative and Flexibility in their Adaption to Telemental Health Nearly all participants shared that they spent much time preparing for telemental health sessions. The preparation was largely self-directed, in both concrete ways (i.e. creating an in-home office space for video calls, paying the internet bill), and non-concrete ways (i.e. a "change in mindset," brainstorming how to engage in telemental health cross-culturally). Two subthemes related to learning and flexibility emerged from the data:

(a) Providers engaged in telemental health learning and sought out resources to acquire new skills. While the state department of behavioral health, DBHDD, provided a mandatory training on telemental health, providers reported several other methods of learning that they either sought out themselves or participated in on a volunteer basis. For example, several providers reported that the internet was a valuable resource for searching for information on telemental health practice. Other providers reported that they benefited from engaging in optional "check-in" meetings with agency supervisors or with colleagues who had telemental health experience. One provider gave credit to her family members, also, stating, "colleagues and family have helped me figure things out." However, refugee providers also reported the need for "more education

Table 2 Telemental health themes among refugee mental health providers and exemplar quotes $(\mathrm{N}=17)$

1. Refugee mental health providers are self-directed and flexible in their adaption to telehealth

1a. Providers engaged in telehealth learning and sought out resources to acquire new skills

"Supervision, ongoing training and resources provided by my agency, and regular check in meetings where support and guidance are given to help improve how I do telehealth services"

"Colleagues and family have helped me figure things out"

"YouTube \& different articles"

"Started using Doxyme \& Zoom. Learned to do 3 way phone calls (interpreters for sessions)"

1b. Providers exhibited flexibility in their daily work routine and their therapeutic approach

"I had to set up an "office" in my room to make it visually acceptable to clients. I had to buy a headset so I can talk to my clients and maintain their confidentiality. I had to purchase lighting to adequately light my office space." "Making a routine where I would not be interrupted to have my sessions, finding a room where privacy is secure and using headphones, explaining to clients the need for and benefits of telehealth services"

"Increased attention to verbal validation of feelings"

2. Providers reported several obstacles to effective telehealth, including refugee-unique obstacles

2a. Client barriers to telehealth are primarily related to few resources and low tech literacy

"Some clients don't pay phone bills"

"Clients not having accessibility to computer/laptop to do evaluations. Clients not having strong enough wifi signal/ data on the phone to have a video session"

"Clients having limited minutes or internet data"

"Many of my clients do not have a private and confidential space"

"Telehealth becomes challenging when working with computer-incompetent clients and clients without computers nor internet"

"Some clients do not know how to video chat"

2b. Language issues and the lack of nonverbals are also obstacles to effective telehealth

"Telehealth with an interpreter (so, 3 people) is trickier than in person"

"Getting interpreters for non-English speaking individuals. Getting the correct translation from interpreter"

"More difficulty hearing and understanding individuals"

"[It is] challenging not seeing body language during sessions"

"Not having nonverbal cues to help me gauge individuals' reactions"

"Working through what the mood of the person is virtually, if I have not previously met with the IND" 
for how to use telemental health services in a culturally competent way."

(b) Providers exhibited flexibility in their daily work routine and their therapeutic approach. The process of adapting to telemental health required many mental health Providers to physically adapt their space in order to have a quiet, uninterrupted, and confidential space for telemental health sessions. For example, one provider shared her experience of "making a routine where I would not be interrupted to have my sessions (and) finding a room where privacy is secure and using headphones." Some providers reported that they had to change their work hours and be more flexible with client sessions due to clients being "hard to reach." For example, while an in-person session might be more rigid in its schedule (i.e. a Tuesday from 5 to $6 \mathrm{pm}$ ), a telemental health session might require several phone calls at different times in order to reach the refugee client.

Providers were also flexible in their therapeutic approach. A few providers shared that they had to adjust their therapeutic approach from a non-directive, person-centered approach to a more directive approach as some clients did not share as freely via telemental health as they did in person. Another provider shared the opposite, reporting that she was "spending more time listening to the client during the call" than she normally would in person. A few providers who were using the phone and not video for sessions reported the need to compensate for the lack of nonverbal interaction, as one provider shared that she demonstrated "increased attention to verbal validation of feelings."

Theme 2: Providers Reported Several Obstacles to Effective Telemental Health, Including Refugee-Unique Obstacles Refugee mental health providers reported several obstacles to effective telemental health, including technological barriers, client hesitance, language issues, and the lack of nonverbal cues. Several of the barriers reported were related to the characteristics of the refugee client, many of whom speak no or minimal English, may have low technological literacy, and often lack financial resources. As a result, there were two subthemes: (1) client barriers to telemental health are primarily related to few resources and low technological literacy, and (2) language issues and the lack of nonverbals are secondary obstacles to effective telemental health.

(a) Client Barriers to Telemental health are Primarily Related to Few Resources and Low Tech Literacy. Refugee mental health providers frequently reported that the lack of access to technological devices (computer, tablet, phone, etc.), internet availability, and/or money to pay for phone minutes or data was a hindrance to providing telemental health for some of their clients. One barrier reported was "clients not having strong enough Wi-Fi signal/data on the phone to have a video session"-indicating that even if the clients have access, they do not have enough bandwidth to engage in video sessions. Additionally, one provider shared, "many of my clients do not have a private and confidential space," which was commonly reported by other refugee providers as well.

Providers also shared that some of their refugee clients had no or little understanding of how to work tech devices (i.e. "some clients do not know how to video chat"), which made telemental health engagement difficult at best. One provider stated, "telehealth becomes challenging when working with computer-incompetent clients and clients without computers nor internet." Another provider shared with exasperation:

Telehealth should be an option for clients based on the clients' strengths and weaknesses. For at-risk clients, they should not have an option to ONLY have telehealth due to the severity of their case; therapists/care managers should decide which delivery service method is appropriate for at risks clients.

(b) Client Hesitance, Language Issues, and the Lack of Nonverbals are Also Obstacles to Effective Telemental Health. Refugee mental health providers shared that they had an especially difficult time building rapport and getting to know and understand their clients whom they met for the first time via telemental health. One provider shared that "working through what the mood of the person is virtually [is difficult] if I have not previously met with the individual." Another provider shared that she was spending time "explaining to clients the need for and benefits of telehealth services." Similarly, providers also reported that not being able to see the client's nonverbal cues was a difficulty they encountered in providing effective telemental health. Providers also shared that finding interpreters for clients via telemental health was more difficult than in person, and even with an interpreter there was "more difficulty hearing and understanding individuals." Additionally, providers shared that incorporating all three persons into telemental health sessions was an obstacle"telehealth with an interpreter (so, 3 people) is trickier than in person." 


\section{Discussion}

Refugee mental health providers in this study described a generally successful process of adaptation to telemental health in the midst of COVID-19 despite encountering various obstacles. In discussing their experiences, participants also shared the changes they made in the process of transitioning to telemental health.

The experiences reported here provide important insights into refugee mental health providers in resettlement areas and telemental health - an area that, to the authors' knowledge, has not been reported in the literature. While the experiences here are similar to those of other mental health providers using telemental health, the participants in this study reported telemental health obstacles and advantages that are unique to the refugee population. For example, the interrelated obstacles of communication struggles (due to not speaking English or speaking limited English), incorporation of a third person in the session (interpreter), low technological literacy (due to lack of technological usage in homeland), and/or lack of access to computers, phones, and/or internet (due to low income as a resettled refugee) are a unique combination of challenges to the refugee mental health provider. We wondered if it was the totality of combined barriers that left service providers feeling overwhelmed or that they were not able to help their clients as much as in-person services.

We also wondered how refugee mental health providers were overcoming the obstacles that they reported. For example, participants reported numerous telemental health barriers, as described above, but they also reported high self-efficacy in telemental health usage. Refugee mental health providers utilize a holistic, flexible framework in their cross-cultural clinical work. This developed skillfulness at adapting clinical practices may explain how the providers were able to overcome telehealth obstacles. More research is needed to understand how refugee-serving mental health providers are adapting specific clinical skills-such as addressing confidentiality, rapport building, assessment, intervention, and managing crisis- to the telemental health format.

This study highlighted how differences in cross-cultural and cross-linguistical communication were amplified in the telemental health, as evidenced by the inability or limited ability to observe non-verbal communication and lag time in verbal communication. Since this study was conducted less than two months following the shift from inperson to telemental health, the research team also questioned how much of the success in telemental health was due to a previously established therapeutic relationship, and whether the refugee mental health providers would be less able to build strong therapeutic relationships and overcome telemental health barriers with new clients.
As mental health agencies grapple with the sudden need for telemental health trainings due to the COVID-19 pandemic, clinical social work practitioners and policymakers must prioritize the integration of skills and competencies of both telemental health and cultural factors (Hilty et al., 2020). For example, the majority of the refugee population are from cultures that utilize a "high context" communication style, which values underlying context, implicit messages, and nonverbal communication such as voice tone, facial expression, gestures, and eye movement (Zwi et al., 2017). If telemental health inherently prioritizes direct verbal communication, what steps can refugee-serving practitioners take to be culturally sensitive to indirect verbal communication styles?

This preliminary study also shed light on future research directions. The COVID-19 pandemic created a unique situation that required the participants in this study to adopt telemental health quickly in order to continue meeting the service needs of their clients. However, the literature suggests that telemental health may not be a good fit for some individuals, diagnoses, or cultures (Luxton et al., 2014). The risk of in-person services during a pandemic should be weighed against the risk of untreated severe mental illness for clients who cannot utilize telemental health, or those for whom the barriers to effective treatment are too significant. Additional research is needed to understand telemental health within the context of COVID-19 and prohibited in-person sessions. There is also a need to understand the benefits of telehealth for the refugee population when they are not able to engage in telemental health "best practices" such as a private, confidential setting with videoconferencing (Luxton et al., 2014; Shore et al., 2018).

\section{Limitations}

There are limitations to this study that should be acknowledged. The small sample size is a limitation, and all participants were recruited from the same clinic. While it is beneficial that all participants reported feeling supported by their employer, the results should be interpreted in light of this highly supportive work environment. The results might be different, and possibly more negative, for mental health providers who are not employed in a highly supportive work environment, or who work in more isolated private practice settings. Additionally, this study did not explicitly ask about any benefits of telemental health.

\section{Conclusion}

As this article concludes, our hope is that the experiences of these seventeen mental health providers enrich existing limited knowledge about telemental health with refugee clients. The participants in this study shared the processes and 
barriers they experienced in adapting to telemental health. Despite reporting several obstacles to effective telemental health practice, providers also shared that they felt equipped to provide telemental health, and the majority of providers believed that their clients were receiving the same benefit from telemental health as in-person sessions.

While there are many known barriers to telemental health, telemental health also provides the opportunity to overcome mental healthcare disparities in refugee communities, such as the potential ability to access a practitioner who speaks the client's language and is from the same or similar cultural background (Stewart et al., 2017), or, of course, the ability to safely access services during a pandemic. At the same time, practitioners should consider whether effective mental health treatment can be provided to each client based on the barriers that may be present.

COVID-19 is one of several growing threats to traditional in-person mental health service. Pandemics, natural disasters, and changes in funding allocations are increasingly pushing health and mental health service delivery into the online realm. This study suggests that practitioners are both capable and in need of resources to successfully adapt to a changing healthcare landscape and meet the needs of diverse populations.

Funding There is no funding to report.

\section{Declarations}

Conflict of interest There are no conflicts of interest or competing interests to report.

Ethical Approval The institutional review board of the University of Georgia approved all procedures.

Consent to Participate All participants were informed about their confidentiality and were asked to voluntarily electronically consent to participate. There was no incentive for participation. "Your involvement in the study is voluntary, and you may choose not to participate or to stop at any time without penalty or loss of benefits to which you are otherwise entitled.'

Consent for Publication "The results of the research study may be published, but your name or any identifying information will not be used."

\section{References}

Almoshmosh, N., Jefee-Bahloul, H., Abdallah, W., \& Barkil-Oteo, A. (2020). Use of store-and-forward tele-mental health for displaced Syrians. Intervention (15718883), 18(1), 66-70

Ashfaq, A., Esmaili, S., Najjar, M., Batool, F., Mukatash, T., Al-Ani, H. A., \& Koga, P. M. (2020). Utilization of mobile mental health services among Syrian refugees and other vulnerable Arab populations-A systematic review. International Journal of Environmental Research and Public Health, 17(4), 1295
Brooks, E., Turvey, C., \& Augusterfer, E. F. (2013). Provider barriers to telemental health: Obstacles overcome, obstacles remaining. Telemedicine and e-Health, 19(6), 433-437

Creswell, J. W. (2012). Qualitative inquiry and research design: Choosing among five approaches. Sage publications.

Cox, A., Lucas, G., Marcu, A., Piano, M., Grosvenor, W., Mold, F., Maguire, R., \& Ream, E. (2017). Cancer survivors' experience with telemental health: A systematic review and thematic synthesis. Journal of Medical Internet Research, 19(1), e11

Fazel, M., Wheeler, J., \& Danesh, J. (2005). Prevalence of serious mental disorder in 7000 refugees resettled in western countries: A systematic review. The Lancet, 365, 1309-1314

Field, M. J. (Ed.). (1996). Telemedicine: A guide to assessing telecommunications for health care. National Academies Press.

Gellis, Z. D., Kenaley, B. L., \& Have, T. T. (2014). Integrated telemental health care for chronic illness and depression in geriatric home care patients: The integrated telemental health education and activation of mood (I-TEAM) study. Journal of the American Geriatrics Society, 62(5), 889-895

Gros, D. F., Lancaster, C. L., López, C. M., \& Acierno, R. (2018). Treatment satisfaction of home-based telehealth versus in-person delivery of prolonged exposure for combat-related PTSD in veterans. Journal of Telemedicine and Telecare, 24(1), 51-55.

Hilty, D. M., Gentry, M. T., McKean, A. J., Cowan, K. E., Lim, R. F., \& Lu, F. G. (2020). Telemental health for rural diverse populations: Telebehavioral and cultural competencies, clinical outcomes and administrative approaches. Mhealth, 6, 20

Jefee-Bahloul, H., Moustafa, M. K., Shebl, F. M., \& Barkil-Oteo, A. (2014). Pilot assessment and survey of Syrian refugees' psychological stress and openness to referral for telepsychiatry (PASSPORT Study). Telemedicine and e-Health, 20(10), 977-979

Kirzinger, A., Kearney, A., Hamel, L., \& Brodie, M. (2020). KFF health tracking poll-Early April 2020: The impact of coronavirus on life in America. Kaiser Family Foundation.

Lamkaddem, M., Stronks, K., Devillé, W. D., Olff, M., Gerritsen, A. A., \& Essink-Bot, M. L. (2014). Course of post-traumatic stress disorder and health care utilisation among resettled refugees in the Netherlands. BMC Psychiatry, 14(1), 90

Levy, C. E., Spooner, H., Lee, J. B., Sonke, J., Myers, K., \& Snow, E. (2018). Telemental health-based creative arts therapy: Transforming mental health and rehabilitation care for rural veterans. The Arts in Psychotherapy, 57, 20-26

Luxton, D. D., Pruitt, L. D., \& Osenbach, J. E. (2014). Best practices for remote psychological assessment via telemental health technologies. Professional Psychology: Research and Practice, $45(1), 27$

North, S. (2020). Addressing students' mental health needs via telemental health. North Carolina Medical Journal, 81(2), 112-113

Perry, K., Gold, S., \& Shearer, E. M. (2020). Identifying and addressing mental health providers' perceived barriers to clinical video telemental health utilization. Journal of Clinical Psychology, 76(6), 1125-1134

Saldaña, J. (2015). The coding manual for qualitative researchers. Sage.

Schulz, T. R., Leder, K., Akinci, I., \& Biggs, B. A. (2015). Improvements in patient care: Videoconferencing to improve access to interpreters during clinical consultations for refugee and immigrant patients. Australian Health Review, 39(4), 395-399

Shore, J. H., Yellowlees, P., Caudill, R., Johnston, B., Turvey, C., Mishkind, M., Krupinski, E., Myers, K., Shore, P., Kaftarian, E., \& Hilty, D. (2018). Best practices in videoconferencing-based telemental health April 2018. Telemedicine and e-Health, 24(11), $827-832$

Soron, T. R., Heanoy, E. Z., \& Udayasankaran, J. G. (2019). Did Bangladesh miss the opportunity to use telepsychiatry in the Rohingya refugee crisis? The Lancet Psychiatry, 6(5), 374 
Standing, C., Standing, S., McDermott, M. L., Gururajan, R., \& Kiani Mavi, R. (2018). The paradoxes of telemental health: A review of the literature 2000-2015. Systems Research and Behavioral Science, 35(1), 90-101

Stewart, R. W., Orengo-Aguayo, R. E., Gilmore, A. K., \& de Arellano, M. (2017). Addressing barriers to care among Hispanic youth: Telemental health delivery of trauma-focused cognitive behavioral therapy. The Behavior Therapist, 40(3), 112

Tuerk, P. W., Yoder, M., Ruggiero, K. J., Gros, D. F., \& Acierno, R. (2010). A pilot study of prolonged exposure therapy for posttraumatic stress disorder delivered via telemental health technology. Journal of Traumatic Stress, 23(1), 116-123

World Health Organization. (2020). Mental health and psychosocial considerations during the COVID-19 outbreak, 18 March 2020 (No. WHO/2019-nCoV/MentalHealth/2020.1). World Health Organization

Zhou, X., Snoswell, C. L., Harding, L. E., Bambling, M., Edirippulige, S., Bai, X., \& Smith, A. C. (2020). The role of telemental health in reducing the mental health burden from COVID-19. Telemedicine and e-Health, 26(4), 377-379

Zwi, K. J., Woodland, L., Kalowski, J., \& Parmeter, J. (2017). The impact of health perceptions and beliefs on access to care for migrants and refugees. Journal of Cultural Diversity, 24(3), 63
Publisher's Note Springer Nature remains neutral with regard to jurisdictional claims in published maps and institutional affiliations.

Lindsey Disney is an Assistant Professor at the University at Albany, State University of New York, School of Social Welfare. Dr. Disney examines research related to mental health disparities. Dr. Disney has worked as a clinical social worker in refugee mental health for the past ten years.

Orion Mowbray is an Associate Professor and Research Director at the University of Georgia, School of Social Work. Dr. Mowbray examines two areas of research: (1) Promoting access to mental health services, and (2) Understanding differential outcomes associated with mental health services utilization.

Dana Evans is the Clinical Director at a refugee-serving mental health clinic in a large refugee resettlement area in Clarkston, Georgia. 